Annuaire suisse de politique de développement

16 | 1997

Environnement et développement, 5 ans après Rio

\title{
7. Coopération avec les pays de l'Europe centrale et orientale et de la CEI
}

\section{(2) OpenEdition \\ Journals}

Édition électronique

URL : http://journals.openedition.org/aspd/793

DOI : 10.4000/aspd.793

ISSN : 1663-9669

Éditeur

Institut de hautes études internationales et du développement

Édition imprimée

Date de publication : 1 mars 1997

Pagination : 171-176

ISSN : 1660-5934

Référence électronique

«7. Coopération avec les pays de l'Europe centrale et orientale et de la CEI », Annuaire suisse de politique de développement [En ligne], 16 | 1997, mis en ligne le 07 août 2012, consulté le 08 septembre 2020. URL : http://journals.openedition.org/aspd/793 ; DOI : https://doi.org/10.4000/aspd.793

(c) The Graduate Institute | Geneva 


\section{COOPÉRATION AVEC LES PAYS DE L'EUROPE CENTRALE ET ORIENTALE ET DE LA CEI}

1,65 milliard de francs sont à disposition pour l'aide aux pays de l'Est. Les principaux instruments de l'aide publique bilatérale sont la coopération technique, l'aide financière, la garantie de crédits. Le Conseil fédéral souhaite, pour orienter la coopération, mieux tenir compte des progrès réalisés dans les processus de réforme dans les différents pays de l'Est. L'aide est en train d'être déplacée des Etats Baltes et des pays de l'Europe centrale vers les pays de l'Europe du Sud-Est et de la CEI. Dès 1996, la gestion de l'aide a été réorganisée dans la Direction pour le développement et la coopération $(D D C)$. L'OFAEE gère l'aide financière, les garanties de crédits, les mesures de promotion du secteur privé, ainsi que l'aide multilatérale (BERD).

L'aide aux pays de l'Est économiquement les plus avancés est répertoriée par le Comité d'Aide au développement non pas dans l'« aide publique au développement » (APD), mais dans un agrégat séparé d' « aide publique ». L'aide aux pays de l'Est les plus défavorisés est comptabilisée dans l'APD (et donc comprise dans les données du chapitre VI). Les pays inclus dans la liste des pays en développement sont les suivants: l'Albanie, l'Ex-Yougoslavie, les Républiques d'Asie centrale, l'Arménie, la Géorgie et l'Azerbaïdjan. Ces pays en développement de l'Europe orientale et de l'Asie centrale sont cependant compris dans les crédits de programme d'aide aux pays de l'Est et couverts par l'arrêté concernant la coopération avec les Etats d'Europe de l'Est. L'aide humanitaire à l'Ex-Yougoslavie et aux pays de l'Europe centrale et orientale est financée par le crédit de programme pour la continuation de l'aide humanitaire internationale.

\section{$\square$ Crédits de programme et organisation}

1650 millions de francs sont à disposition pour financer l'aide publique aux pays de 1'Est. Le premier crédit-cadre adopté en 1990 portait sur un montant de 250 millions de francs. Il était en faveur de la Hongrie, de la Pologne et de l'ex-Tchécoslovaquie. Le deuxième crédit de programme de 800 millions de francs, adopté en 1992, permettait d'ouvrir l'aide à l'Albanie, la Bulgarie, les Etats Baltes, la Macédoine, la Roumanie et la Slovénie. Ce crédit a été augmenté de 600 millions de francs en mars 1993 pour l'étendre à tous les pays issus des anciennes républiques soviétiques (CEI, Communauté des Etats indépendants). Sur ce deuxième crédit cadre d'un montant total de 1,4 milliard de francs, les engagements s'élevaient à 1,1 milliard de francs à la fin de 1995. La préparation du message concernant le troisième crédit de programme a débuté.

L'arrêté fédéral de portée générale concernant la coopération avec les Etats de l'Europe de l'Est constitue la base légale pour les relations avec ces Etats. Il a été adopté au Parlement en 1995, mais n'est pas encore en vigueur (à fin décembre 1996) Cf Annuaire 1996, p.177 pour les débats au Conseil National. Cet arrêté fédéral a une durée de 10 ans, soulignant ainsi le caractère transitoire de la coopération avec ces pays. Il fixe les objectifs principaux de la coopération 
avec les pays de 1'Est : promotion de l'Etat de droit, de la démocratie et la transition vers l'économie de marché.

\section{$\square$ Organisation de la gestion de l'aide et réorientation de la coopération}

L'assistance technique et la coordination de la coopération avec les pays de l'Est étaient jusqu'en 1995 assumées par le Bureau de la coopération avec l'Europe centrale et orientale, créé dans ce but et rattaché à la Division politique $\mathrm{I}$ du Département fédéral des affaires étrangères. L'aide suisse à l'Europe de l'Est s'est donc construite au départ sans se baser vraiment sur l'expérience acquise par la Direction de la coopération au développement (DDA) pour l'aide aux pays du Sud. Dès 1996, la coopération avec pays de l'Est a été rattachée avec celle destinée aux pays en développement dans la structure qui s'appelle désormais Direction pour le développement et la coopération (DDC). L'ancienne subdivision en secteurs est remplacée par trois sections géographiques à la DDC : Europe centrale, Europe du Sud-Est et CEI (Communauté des Etats Indépendants). On relèvera que la coordination de l'aide est assurée par la DDC, mais que le $45 \%$ des fonds d'aide sont versés par l'OFAEE. L'OFAEE gère l'aide financière et les mesures économiques, dans la division pour le soutien économique de l'Europe centrale et orientale.' Trois sections de l'OFAEE s'occupent de ce soutien : aide financière à l'Europe centrale et orientale, aide financière aux Etats de la CEI (pour l'aide financière et les garanties de crédit), et la section promotion du secteur privé/BERD/information.

Conformément à ce qui avait été proposé lors de l'adoption de l'augmentation du deuxième crédit-cadre en 1993, le Conseil fédéral publie un Rapport annuel sur la coopération avec l'Europe de l'Est pour informer les commissions parlementaires concernées sur les projets adoptés et donner une évaluation de la coopération. L'arrêté fédéral concernant la coopération avec les pays de l'Est prévoit l'instauration d'une commission consultative chargée de donner au Conseil fédéral son avis sur les objectifs et les priorités de l'aide, ainsi que d'examiner les résultats des évaluations. Cette commission n'est pas encore opérationnelle.

La commission de gestion du Conseil national avait soumis la coopération de la Confédération avec les pays de l'Est à une évaluation critique. L'un des problèmes évoqués lors d'évaluation et lors des discussions au Parlement sur l'arrêté fédéral était la trop grande dispersion sectorielle et géographique de l'assistance technique. Les secteurs prioritaires sont différents d'un pays à l'autre, selon le rythme de la transition économique, variable d'un pays à l'autre. Le processus de réforme différenciée dans les pays de l'Est a conduit à de nouvelles orientations de l'aide. Certains pays d'Europe centrale, dont la Tchéquie, la Pologne ou la Hongrie, enregistrent des progrès rapides dans leur transition vers l'économie de marché. Les pays nordiques concentrent leur aide vers les Etats baltes. Le processus de transition est en revanche beaucoup plus lent en Europe du Sud-Est et les problèmes économiques restent graves dans la Communauté des Etats Indépendants (CEI). C'est dans ces pays que la Suisse souhaite progressivement concentrer l'aide publique.

${ }^{1}$ OFAEE. Service du soutien économique aux pays d'Europe centrale et orientale. Effingerstrasse 1-3, 3003 Berne. 


\section{$\square$ Répartition géographique et par instrument de l'aide publique pour les pays de l'Est 1995}

Le tableau 25 résume les principales contributions de la Confédération par pays bénéficiaire et par instrument (sans les garanties de crédits). La coopération technique s'est élevée à 50 millions de francs en 1995 (54 millions en 1994), l'aide financière à 66 millions de francs (95 millions en 1994) et l'aide humanitaire à 41 millions de francs (40 millions en 1994). A cette aide s'ajoutent les mesures de promotion du commerce (3,6 millions de francs en 1995), l'aide aux investissements (11,5 millions de francs).

\section{$\square$ Formes de coopération}

\section{Coopération technique}

Les principaux pays bénéficiaires de la coopération technique étaient en 1995 la Hongrie, la Russie et le Kirghizistan. La DDC souhaite une concentration progressive de la coopération technique sur ces pays de l'Europe du Sud-Est et de la CEI. L'Albanie, la Bulgarie, la Macédoine et la Roumanie deviennent les pays de concentration de l'aide. Les programmes spéciaux en Russie et au Kirghizistan seront poursuivis et de nouveaux programmes spéciaux sont en préparation pour l'Ukraine et la Bosnie. Les secteurs prioritaires dans la coopération technique sont la formation, l'agriculture, l'environnement, la santé publique, ainsi que le soutien aux administrations communales. Une grande partie des projets de formation concernent le perfectionnement professionnel de cadres et entrepreneurs de petites et moyennes entreprises ou de banques. La formation d'enseignants du niveau secondaire est aussi soutenue dans plusieurs pays.

\section{Coopération financière}

La coopération financière permet de financer des projets pour lesquels l'économie suisse est en mesure de fournir, à des conditions avantageuses, des biens d'équipement ou des prestations de services. Elle se compose de l'aide financière, de l'aide à la balance des paiements, des mesures de désendettement, des garanties de crédits et de la promotion des investissements et du commerce.

Aide financière : L'aide financière permet notamment de soutenir des investissements dans les infrastructures de base, de l'équipement dans le domaine de la santé et de l'environnement. Elle est octroyée pour financer des investissements qui ne génèrent pas un rendement financier suffisant pour pouvoir accorder un crédit aux conditions du marché. L'OFAEE étudie la faisabilité technique, financière et économique des projets, ainsi que leur impact sur l'environnement.

Parmi les nouveaux projets décidés en 1995, on trouve par exemple la modernisation du métro et des trams à Bucarest (Roumanie), la livraison de biens d'équipement et de services pour la construction d'une station d'épuration et de traitement d'eau potable en Hongrie, en Estonie et en Tchéquie, la livraison d'équipements médicaux en Tchéquie et en Russie. L'aide financière versée à l'Albanie est accordée surtout sous forme de cofinancements de projets des banques de développement (BERD, AID). En 1996, des aides financières ont par exemple été octroyées pour la remise en état d'une centrale hydroélectrique en Bosnie, la livraison d'une station électrique en Albanie, la rénovation de l'infrastructure de stérilisation et de désinfection dans des hôpitaux estoniens, des équipements médicaux pour Bélarus, la livraison d'équipements pour le traitement de l'eau potable en Russie. 
Tableau no 25

Coopération avec les pays de l'Europe centrale et orientale

\begin{tabular}{|c|c|c|c|c|}
\hline \multicolumn{5}{|c|}{ Versements en 1995 par pays (en milliers de francs) } \\
\hline$\overline{\text { Pays }}$ & $\begin{array}{r}\text { Coopération } \\
\text { technique }\end{array}$ & $\begin{array}{r}\text { Aide } \\
\text { financière }\end{array}$ & $\begin{array}{r}\text { Aide } \\
\text { humanitaire }\end{array}$ & Total \\
\hline Ex-Yougoslavie*, divers Etats & & & $30 ’ 675$ & $30 ` 675$ \\
\hline Albanie* & 4’025 & 9’265 & 541 & $13{ }^{\prime} 831$ \\
\hline Russie & 6’918 & $1 ' 533$ & 5,321 & $13^{\prime} 772$ \\
\hline Kirghizistan/Kirghisie* & $5^{\prime} 111$ & 4’306 & 107 & $99^{\prime} 524$ \\
\hline Bulgarie & 3’175 & 5’396 & 110 & $8 ’ 682$ \\
\hline Pologne & 2'660 & 6’000 & & $8^{\prime} 660$ \\
\hline Hongrie & 7’021 & $1 ' 500$ & & $88^{\prime} 521$ \\
\hline Roumanie & 3'804 & 3’403 & 121 & 7’328 \\
\hline Bosnie* & $1 ' 600$ & $4^{\prime} 900$ & & $6^{\prime} 500$ \\
\hline Slovaquie & $1 ' 760$ & $4{ }^{\prime} 200$ & & 5,960 \\
\hline Tchéquie & 715 & 4'900 & & 5,615 \\
\hline Lettonie & 434 & 3'795 & & $4 ' 229$ \\
\hline Macédoine* & 473 & 3'605 & & $4^{\prime} 078$ \\
\hline Estonie & 891 & 2'327 & & 3,218 \\
\hline Lituanie & 824 & 1'155 & & 1'979 \\
\hline Géorgie* & & & 1'898 & 1'898 \\
\hline Slovénie* & 1'302 & & & $1 ' 302$ \\
\hline Tadjikistan* & & & 863 & 863 \\
\hline Azerbaïdjan* & & & 847 & 847 \\
\hline Bélarus/Biélorussie & & 407 & 354 & 761 \\
\hline Ouzbékistan* & & & 546 & 546 \\
\hline Ukraine & & 283 & 28 & 311 \\
\hline Arménie* & & & 42 & 42 \\
\hline projets régionaux & 6'807 & 3'308 & & $10 ’ 115$ \\
\hline Administration & 2'479 & $5 ' 439$ & & $7^{\prime} 918$ \\
\hline Total** & 49'998 & $65 ' 723$ & 41'453 & 157'17 \\
\hline
\end{tabular}

Remarques :

*Ces pays sont compris dans la liste des pays en développement.

** Les chiffres du tableau ne contiennent pas la promotion du commerce et des investissements, ni les garanties de crédits.

Sources : Rapport annuel du Conseil fédéral sur la coopération avec les pays d'Europe centrale et orientale 1995, Berne, septembre 1996 DDC/DEZA, Humanitäre Hilfe Statistiken, OFAEE/BAWI.

En 1995, les principaux pays bénéficiaires de l'aide financière étaient l'Albanie, la Pologne, la Bulgarie, la Tchéquie et la Bosnie. Les nouveaux engagements d'aide financière ce concentrent dès 1995/1996 davantage sur les pays du Sud- 
Est de l'Europe (Albanie, Bulgarie, Roumanie et Macédoine). Dans les pays plus avancés, l'accent sera donné au soutien au secteur privé.

Garantie de crédits : Ces garanties permettent l'accès à des crédits commerciaux destinés à financer des livraisons de biens d'équipement prioritaires et des projets dont on attend un rendement suffisant pour payer le remboursement et les intérêts. Cet instrument permet d'assumer les risques liés à l'octroi de garanties à des pays n'ayant pas accès à la Garantie des risques à l'exportation (GRE). La situation économique de certains pays est si difficile qu'elle représente des risques trop élevés pour l'engagement de la GRE (par exemple dans les pays de la CEI, l'Albanie, la Croatie ou la Macédoine). La Confédération offre par exemple des garanties de crédit pour les exportations vers les Etats baltes qui ne sont pas assurés par la GRE. La substitution de la garantie de crédit par la GRE est progressive, en tenant compte de l'évolution économique favorable dans certains pays et de la privatisation du secteur bancaire. Certaines banques privées commencent ainsi à garantir la couverture du risque de ducroire privé, en Russie et Lituanie par exemple.

\section{$\square$ Coopération régionale et aide transitant par les organisations multilatérales}

La Suisse a financé des stages organisés par l'OMC pour des représentants des pays en transition. Elle soutient aussi des projets du CCI : mise en œuvre des accords de l'Uruguay Round, compétitivité des PME dans l'exportation, information commerciale, promotion des exportations des entreprises polonaises du secteur des machines.

Banque européenne pour la reconstruction et le développement (BERD)

La Suisse est membre de la BERD depuis sa création et détient $2,28 \%$ du capital-actions de la banque (soit une part suisse de 410 millions de francs). La BERD soutient des projets dans le secteur privé (appui à la création de PME et au secteur financier), ainsi que des projets d'infrastructure et de protection de l'environnement. Depuis son ouverture en 1991, la BERD a engagé dans les pays de l'Est environ 10 milliards de francs suisses dans plus de 300 projets (garanties, prêts, prises de participation, fonds régionaux de capital-risque etc.). $\mathrm{Ne}$ pouvant pas financer toute seule des actions très coûteuses, la Suisse souhaite accroître l'efficacité de l'aide en s'associant à des actions internationales par le biais des cofinancements avec la Banque européenne. La Suisse prévoit de participer à la première augmentation du capital de la BERD (1997). Un Message sera présenté aux Chambres fédérales pour la participation de la Suisse. La Suisse poursuit sa collaboration avec le Programme de sécurité nucléaire géré par la BERD.

\section{Trusts Funds}

La Suisse soutient des trusts Funds dans différentes organisations multilatérales de financement (Banque Mondiale, BERD, Société financière internationale SFI). Ces fonds permettent de financer des études de faisabilité dans des secteurs clés (en autres énergie, transports, environnement, santé). Cet instrument permet de créer des synergies avec certains projets bilatéraux et offre des opportunités pour l'industrie d'exportation de biens et services suisses. Au minimum $50 \%$ des moyens financiers sont, en effet, liés à l'engagement de consultants suisses. 4,9 et respectivement 1 million de francs ont été engagés par la Suisse en 1995 pour 
la reconstitution de ces fonds auprès de la Banque mondiale et la BERD pour les années à venir. 950 '000 francs ont été engagés la même année pour la reconstitution du Trust Fund de la Banque Mondiale "Environnement pour l'Europe » (identification de projets d'investissement dans l'environnement). Un autre Trust Fund multilatéral est ouvert à la Banque Mondiale pour la réalisation de projets de réhabilitation d'infrastructures de base et pour des PME en Bosnie. La contribution de la Suisse à ce fonds s'est élevée à 6,9 millions de francs pour 1996.

\section{Aide des cantons et communes}

L'aide des cantons suisses versée aux pays de l'Est s'est élevée à 1,7 million de francs en 1995 (1,1 million de francs en 1994) et l'aide des communes aussi à 1,7 million de francs en 1995 (1,6 en 1994). Près de la moitié de cette aide est gérée directement par les cantons et communes, sans passer par les institutions privées de coopération.

\section{Aide privée pour les pays de l'Europe de l'Est}

L'aide fournie par les institutions privées pour les pays de l'Europe de l'Est s'est élevée à 14,5 millions de francs en 1995, sans compter les pays en développement de l'Est. Ce montant est assez stable ces dernières années. Les principaux pays destinataires des 10,6 millions de francs de coopération au développement sont la Roumanie (6,9 millions), et loin derrière, la Russie et la Hongrie $(0,8$ million pour chacun des deux pays). La Roumanie et la Russie ont bénéficié de 83\% des versements d'aide humanitaire.

\section{$\square$ Autres mesures de politique extérieure}

L'aide publique au développement n'est bien sûr qu'un élément de la politique économique extérieure. Cette politique avec les pays de l'Est comprend aussi les visites de délégations économiques, les accords de libre-échange, les accords bilatéraux de commerce et de coopération, les accords de protection des investissements et de double imposition

1997 Diplomatie commerciale et accords économiques bilatéraux, p. 128.

\footnotetext{
SOURCES

Conseil fédéral, Coopération de la Confédération avec l'Europe de l'Est. Rapport annuel 1995, Berne, septembre 1996. OFAEE, Coopération économique avec les pays d'Europe centrale et orientale (crédits de programme I et II, projets pays par pays et projets régionaux), Service du soutien économique aux pays d'Europe centrale et orientale, Berne : OFAEE, 27 novembre 1996.

Conseil fédéral, Rapport sur la politique économique extérieure $95 / 1+2$ et Messages concernant des accords économiques internationaux, du 17 janvier 1996 (Message concernant l'Accord entre les Etats de l'AELE et la Slovénie, Messagẹ concernant les accords de commerce et de coopération économique conclus entre la Confédération suisse et, respectivement, l'Ukraine, la République de Moldova, la République d'Albanie et la Macédoine), in Feuille fédérale FF, vol.1, $\mathrm{n}^{\circ}$ 10, 12 mars 1996, p. 617-949 (peut être commandé à l'adresse suivant : EDMZ, 3003 Bern, Message № 95.091). Bulliard, Pascal, « Coopération suisse avec l’Est », in Vers un développement solidaire, № 135, septembre 1996.

Der Bund, 19. Juni 1996 « Gewicht nach Südosteuropa verlagert ».

Neue Zürcher Zeitung, 31. Mai 1996, 3./4. August 1996.
} 